\title{
Service Class Mapping Based on Integer Programming Algorithm in the Third Party Agent
}

\author{
Bozidar J. Mali, Nemanja M. Ninkovic, Mirjana D. Stojanovic and Gordana I. Savic
}

\begin{abstract}
In this paper, we propose a novel algorithm for mapping of service classes among multiple Internet providers on an end-to-end (E2E) path. The third-party (3P) approach is assumed for E2E service negotiation, whereas the foundation for class mapping is laid on the integer programming mathematical model. The algorithm selects service classes in domains on the path so that requirements for E2E quality of service $(\mathrm{QoS})$ are fulfilled. This selection is based on multiple constraints, referring to performance fulfillment and at the same time aiming to achieve minimal E2E interconnection cost through definition of a single objective function. Performance evaluation has clearly indicated benefits of the proposed algorithm in terms of QoS achievement and decreasing costs, as well as its suitability for services that require stringent $\mathrm{QoS}$ guarantees.
\end{abstract}

Keywords - Integer programming, Mapping, Negotiation, Quality of service, Third party model.

\section{INTRODUCTION}

$\mathrm{S}_{\mathrm{o}}$ OLUTIONS for the future Internet impose achievement of end-to-end (E2E) quality of service (QoS) due to proliferation of users, applications, and new services [1]. So far, intra-domain QoS deployment has proven to be feasible, allowing heterogeneous traffic with various requirements to use a single network infrastructure. However, inter-domain QoS solutions are still a challenging task, because all involved providers have to negotiate and assure service performance in different scenarios in which the offered service traverses outside provider's boundaries. Service providers are commercial entities whose business goals are mainly defined in terms of profit and competitiveness. Besides, heterogeneity of

Paper received March 25, 2015; revised Jun 18, 2015; accepted Jun 20, 2015. Date of publication July 15, 2015. The associate editor coordinating the review of this manuscript and approving it for publication was Prof. Grozdan Petrović.

This paper is a revised and expanded version of the paper presented at the 22th Telecommunications Forum TELFOR 2014.

This research was partially funded by grants (No. TR 32025 and No. III44007) from the Ministry of Education, Science and Technological Development of Serbia.

Bozidar J. Mali and Gordana I. Savic are with the Faculty of Organizational Sciences, University of Belgrade, Serbia (e-mail: malibozidar@gmail.com, goca@fon.bg.ac.rs).

Nemanja M. Ninkovic is with the School of Electrical Engineering, University of Belgrade, Serbia (e-mail:nemanja.ninkovic@amres.ac.rs).

Mirjana D. Stojanovic is with the Faculty of Transport and Traffic Engineering, University of Belgrade, Serbia (e-mail: m.stojanovic@sf.bg.ac.rs). network technologies and intra-domain QoS solutions increase the complexity of E2E service negotiation and management.

In today's Internet, the basic bilateral model [2] is prevalent for the inter-provider QoS delivery. It assumes that only neighboring providers negotiate and establish bilateral service level agreements (SLAs). E2E SLA over a path consisting of multiple domains can be formed by concatenating the existing bilateral agreements. This impedes dynamic E2E service negotiation and management, or even makes it impossible.

Several other models have been considered to deliver inter-provider QoS [3], [4], [5]. Thus, an extension of the basic bilateral approach (also called "a cascade model") enables a single provider to establish and control E2E service by means of an appropriate signaling protocol. Another approach is known as "cooperative", and assumes that several providers should agree on certain baselines needed to fulfill E2E QoS requirements, e.g., service specifications, SLA monitoring, providers' responsibilities in delivering QoS, etc.

The third party (3P) approach assumes that an independent, trusted entity ( $3 \mathrm{P}$ agent) manages interprovider QoS and business processes among a group of domains that are under its authority. There is no need for cooperation among providers or reengineering of their networks. This facilitates fulfillment of E2E QoS requirements, particularly in heterogeneous, mobile and highly competitive internetworking environments.

This paper addresses the problem concerning mapping of service classes among domains on the E2E path. Mapping is needed to overcome different specifications of service classes in different intra-domain QoS architectures. We propose and investigate a novel mapping algorithm, which utilizes benefits of the centralized negotiation via 3P-agent and the integer programming mathematical model (IPMM). The algorithm is based on the 3P agent's awareness of the E2E path, and providers' offers for service classes, in terms of QoS and interconnection costs. Accordingly, 3P agent is able to select the most appropriate class in each domain so that QoS requirements are fulfilled and the interconnection costs are minimized. In further text we refer to the proposed algorithm as 3PIP (3P-based Integer Programming algorithm).

The rest of the paper is organized as follows. Section II surveys the related work. In Section III we present the $3 \mathrm{P}$ model and formulate the service class mapping problem. 
The proposed 3PIP mapping algorithm is explained in Section IV. Evaluation of the algorithm performance is presented in Section V. Section VI concludes the paper.

\section{RELATED WORK}

The absence of universally defined E2E service classes appears as a consequence of heterogeneous QoS standards for different access and core network technologies. Moreover, providers are often allowed to select options for specification of service classes. An example is the de facto QoS standard for core networks - Differentiated Services (DiffServ), which offers high flexibility in the definition of service classes and their associated parameters.

Some authors state that a set of E2E classes well known by all the domains is needed, in order to define a converged policy infrastructure and avoid class mapping [6]. Each domain should follow its own strategy to implement that set of classes. However, such an approach is far from realistic in the highly heterogeneous Internet. The ITU-T recommendation Y.1541 specifies network performance objectives for IP-based services, and defines six network classes and their associated parameters [7].

On the other side, mapping of service classes introduces at least two problems. First, it is difficult to select a set of per-domain classes that should provide a perfect match with E2E QoS requirements. This leads either to resource wasting or decreasing E2E performance assurance. Another problem concerns fairness among providers in resource consuming and fulfilling business objectives.

Static mapping, which assumes predefined relations between service classes in different domains regardless of the current network conditions, has been proposed in [8]. The concept of meta-QoS-class, recommended in [2], provides the boundaries of $\mathrm{QoS}$ parameter values that two classes in adjacent providers' networks must satisfy in order to build a bilateral SLA.

Conformance Matching Scheme (CMS) is a 3P-based algorithm for mapping of service classes on E2E path, starting from E2E QoS requirements [9]. The CMS is performed in the following two steps: (1) calculation of per-domain QoS parameters' boundary values, i.e., performance impairment budgets and (2) selection of the most appropriate class in each domain by determining the closest compliance between the required and the offered QoS level.

The algorithm has been improved further in [10] towards the Policy-based CMS (P-CMS), which enables selection of an appropriate policy for allocating perdomain performance impairment budgets. The P-CMS is well suited to both 3P and cascade models. In the latter case, it uses a signaled-based approach [11] to distribute per-domain QoS requests from the initiating provider to all providers on E2E path.

However, both the CMS and the P-CMS select perdomain classes relying only on performance metrics like delay, jitter, and packet loss rate (PLR). The motivation for this work is to propose and investigate a multiconstraint approach to per-domain class selection, which aims to minimize overall interconnection costs while fulfilling E2E QoS requirements.

\section{A Model of Service Negotiation Via 3P Agent AND PROBLEM FORMULATION}

E2E service negotiation in the 3P model is based on the presence of a trusted 3P agent, which performs an impartial mediation between domains. Advantage of such a centralized approach is particularly expressed in multidomain scenarios as QoS signaling occurs between the 3P agent and each domain, without cooperation between domains.

Domains are independent administrative entities and they retain control of their infrastructure, i.e. routing, service class specifications, interconnection costs definition, etc. E2E services are negotiated via a QoS manager (QM), a per-domain entity that is also responsible for QoS management at the domain level. Based on the domains' offers in terms of service classes, 3P agent selects a single class per each domain according to the implemented mapping scheme. A high level overview of establishing E2E service via a $3 \mathrm{P}$ agent is depicted in Fig. 1.

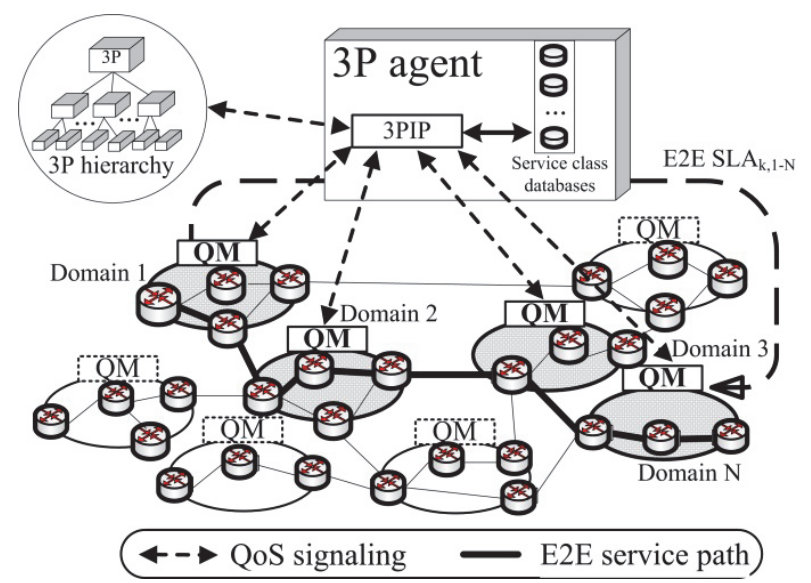

Fig. 1. High level overview of 3P negotiation model.

Aside from the prospect of performing efficient class mapping, 3P model significantly reduces signaling traffic during the process of E2E service negotiation compared to its cascade counterpart [12]. Further improvements delivered by the 3P model are reflected in a predictable service negotiation time and flexibility in the choice of signaling protocol.

Since signaling is path-decoupled, which means that signaling information does not follow paths that are tied to the data messages, standard and proprietary protocols for dynamic service negotiation are applicable [13], [14]. Alternatively, communication between the 3P agent and QM entities can be performed by common management protocols like Simple Network Management Protocol (SNMP) and Common Open Policy Service (COPS). Scalability of the model can be improved through a hierarchical organization of 3P agents [9], [12].

For the purpose of class mapping implementation, 3P agent has databases for each domain, containing detailed information about service classes, routing information, pricing, etc. This information is used as an input for class selection in separate domains when E2E service requirements are specified by the initiating domain (e.g. 
Domain 1 depicted in Fig. 1).

As a trade-off between technical capabilities, market competition and business objectives, domain on the E2E path implements a specific level of service differentiation in terms of offered service classes. Assuming that E2E path consists of $N$ domains, domain $d(d \in\{1,2, \ldots, N\})$ offers $K^{d}$ service classes. For the sake of simplicity, we will adopt common notation $k$ for the selected service class, bearing in mind class mapping among domains, i.e., $k=k_{d}$. E2E service request and service class specification are defined using performance metrics $\mu_{j}, j \in\{1,2, \ldots, M\}$. We adopt the notation $S L A_{k, 1-N}$ for E2E agreement that the initiating domain 1 establishes with the domain $N$, for its service class $k\left(k \in\left\{1,2, \ldots, K^{1}\right\}\right)$. Profit $\pi_{k}$ of the domain 1 , gained on the basis of $S L A_{k, 1-N}$, is:

$$
\begin{gathered}
\pi_{k}\left(\mathrm{SLA}_{k, 1-N}\right)=p_{k}-C_{k}\left(\mathrm{SLA}_{k, 1-N}\right)- \\
-C_{3 P, k}\left(\mathrm{SLA}_{k, 1-N}\right)-C_{c t x, k},
\end{gathered}
$$

where $p_{k}$ is the retail price per bandwidth unit for service class $k, C_{k}\left(S L A_{k, 1-N}\right)$ refers to the overall (E2E) interconnection cost, $C_{3 P, k}\left(S L A_{k, 1-N}\right)$ accounts for $3 \mathrm{P}$ agent's share for E2E service negotiation function, whereas $C_{c t x, k}$ represents service context costs incurred for service maintenance and additional benefits offered by the initiating domain to the end user.

We subsequently propose a 3PIP mapping algorithm that selects service classes conforming to the requested E2E performance and, at the same time, increases projected profit $\pi_{k}$ by minimizing the $C_{k}\left(S L A_{k, 1-N}\right)$ cost.

\section{3PIP SERVICE Class MapPING AlgORITHM}

Prior to proposing a mapping scheme, we need to identify requirements that should be addressed, namely performance metrics and interconnection costs. The 3PIP algorithm relies on the premise that there is a single objective that needs to be accomplished, while simultaneously adhering to multiple constraints [15]. Constraints refer to fulfillment of QoS requirements, whereas the objective function states that E2E interconnection cost for every class mapping obeying the constraints is minimal. Accordingly, the 3PIP is suitable to be used as a selection algorithm for services that have strict performance requirements.

Service differentiation level and posed performance requirements may result in scenarios when the 3PIP is not able to provide class mapping, which is directly related to coarse QoS granularity or inadequately posed service requirements. However, this is not considered as a weakness of the proposed approach as it is a direct consequence of using a mathematical model that yields an exact solution, i.e. class selection aiming to satisfy posed E2E performance limitations.

The 3PIP is based on IPMM [16], an optimization method solvable in polynomial time. For the purpose of class mapping, this mathematical model is applied according to the guidelines in [17]. Bearing in mind recommendations about service differentiation stated in [8] and realistic multi-domain scenarios [11], the 3PIP is a sufficiently scalable mapping scheme.

We introduce variables $y_{d}$ and $x_{k}{ }^{d}(d \in\{1,2, \ldots, N\}$ and

$$
\begin{gathered}
\left.k \in\left\{1,2, \ldots, K^{d}\right\}\right): \\
y_{d}=\left\{\begin{array}{l}
1, \text { if domain } d \text { is on the path } \\
0, \text { if domain } d \text { is not on the path }
\end{array}\right. \\
x_{k}^{d}=\left\{\begin{array}{l}
1, \text { if class } k \text { in the domain } d \text { is selected } \\
0, \text { if class } k \text { in the domain } d \text { is not selected }
\end{array}\right.
\end{gathered}
$$

The 3P agent selects a single class $k$ for which $x_{k}^{d}=1$. As stated before, separate domains define cost per bandwidth unit $c_{k}{ }^{d}$ for each service class. Each class is specified in terms of delay $\mu_{1, k}^{d}$, delay variation (jitter) $\mu_{2, k}^{d}$ and PLR $\mu_{3, k}^{d}$. Correspondingly, E2E service request is defined for delay, jitter and PLR using $\mu_{1, \text { req }}, \mu_{2, \text { req }}, \mu_{3, \text { req }}$, respectively, depending on specific requirements, i.e. some services do not require all performance metrics. Specification of E2E performance requests actually determines boundary values for individual QoS parameters. These values are contained in the SLA for the specific E2E service and penalties should be incurred if performance is degraded in any way.

Composition of metrics is used to determine E2E delay, jitter and PLR from offered values per service class in individual domains. Accordingly, delay and jitter are considered as additive metrics [18], whereas PLR is transformed from indirectly multiplicative into an additive metric according to (6).

We further define constraints in terms of delay, jitter and PLR, depending on the E2E performance requirements, according to the following relations:

$$
\begin{gathered}
\sum_{d=1}^{N} \sum_{k=1}^{K^{d}} \mu_{1, k}^{d} \cdot x_{k}^{d} \cdot y_{d} \leq \mu_{1, \text { req },} \\
\sum_{d=1}^{N} \sum_{k=1}^{K^{d}} \mu_{2, k}^{d} \cdot x_{k}^{d} \cdot y_{d} \leq \mu_{2, \text { req }}, \\
\sum_{d=1}^{N} \sum_{k=1}^{K^{d}}\left(-\log \left(1-\mu_{3, k}^{d}\right)\right) \cdot x_{k}^{d} \cdot y_{d} \leq-\log \left(1-\mu_{3, \text { req }}\right) .
\end{gathered}
$$

Once constraints have been defined, the 3PIP selects conformant classes and further defines the objective function aiming to minimize E2E interconnection cost:

$$
C_{k}\left(\operatorname{SLA}_{k, 1-N}\right)=\min \left\{\sum_{d=1}^{N} \sum_{k=1}^{K^{d}} c_{k}^{d} \cdot x_{k}^{d} \cdot y_{d}\right\} .
$$

Minimization of interconnection cost $C_{k}\left(S L A_{k, 1-N}\right)$ results in an increase of projected profit $\pi_{k}\left(\mathrm{SLA}_{k, 1-N}\right)$, as stated in (1).

We further formulate 3PIP algorithm in the following three steps:

Step 1. E2E performance requirements and service class specification in separate domains on the path are initialized.

Step 2. Service class selection is performed via a branchand-bound method for solving integer programming problems [18] according to the objective function defined in (7) and constraints formulated in (4), (5) and (6).

Step 3. If there are multiple mappings yielding identical cost, mapping that provides the best performance is selected. Selection that offers the highest value of $\sum_{j=1}^{3}\left(\mu_{j, r e q} / \sum_{d=1}^{N} \mu_{j, k}^{d}\right)$ denoting superior performance is selected as a final solution. 
Potential prioritization of separate metrics may be applied, however, for most practical uses Step 3 is a "tie-breaker".

\section{V.Evaluation OF THE 3PIP PERFORMANCE}

In order to evaluate 3PIP class selection, we investigate E2E service performance and cost, which is quantified using fictive monetary units (MU). Mappings are then compared with the existing 3P mapping technique P-CMS that is proposed and analyzed in [10]. The compared algorithms have been implemented in MATLAB.

The following mayor differences exist between the 3PIP and P-CMS that ultimately affect class selection: (1) The P-CMS selects classes in order to achieve as close E2E performance matching as possible. This means that performed mapping may provide metrics values below the requested ones. On the other hand, the 3PIP delivers mapping that is focused towards fulfillment of E2E requirements for each QoS parameter. Consequently, if domains on the path are unable to offer a requested performance, the 3PIP is also unable to perform mapping due to its exact approach; (2) The P-CMS accounts for impairment budgets [13] allocated to each domain on the path. It performs mapping by calculating per-domain performance requests. Conversely, the 3PIP performs mapping solely on the basis of providers' offers, according to the impairment accumulation approach from [11]; (3) The P-CMS mapping process is completely unaware of interconnection costs, whereas the 3PIP is a cost-aware approach.

A primary indicator for class mapping evaluation is the degree of correspondence $(D C)$, which describes the level of conformance to service request [9]. $D C$ parameter is defined as the ratio of requested and offered metric values. The level of conformance is distinguished by observing $D C$ values as follows: (1) $D C>1$ if mapping results in over-engineered E2E performance; (2) $D C<1$ if mapping fails to provide a requested performance and (3) $D C=1$ denotes a perfect match of the requested and offered QoS. In this work, the notation $D C_{j}^{\mathrm{E} 2 \mathrm{E}}$ denotes degree of correspondence defined on E2E basis, per performance metric $j$. Taking into account that delay and jitter are additive, whereas PLR as indirectly multiplicative metric, $D C_{j}^{\mathrm{E} 2 \mathrm{E}}$ is calculated as follows:

$$
\begin{gathered}
D C_{j}^{E 2 E}=\frac{\mu_{j, \text { req }}}{\mu_{j, \text { off }}}=\frac{\mu_{j, \text { req }}}{\sum_{d=1}^{N} \mu_{j, k}^{d}}, \quad \mathrm{j}=1,2, \\
D C_{j}^{E 2 E}=\frac{\left|\log \mu_{j, \text { off }}\right|}{\left|\log \mu_{j, \text { req }}\right|}, \quad j=3,
\end{gathered}
$$

where $\mu_{j, \text { off }}$ denotes offered E2E performance metric.

Testing and comparison are carried out by observing different service requests and service class performance with cost specifications in Table 1 and Table 2, respectively. Service class specifications are given with respect to domain hierarchy, whereas the number of classes per domain and interconnection costs depends on provider's scale. Also, the number of defined classes and metric specifications per class depend on the provider's policy, so various levels of QoS granularity may be expected on the E2E path. Similar QoS specification is used in [9], [10] and [20]. Class mapping is investigated on the two paths consisting of five and three domains respectively, i.e., A1-R1-C-R2-A2 and A3-R3-A4 according to Table 2 . Taking into account domain hierarchy in the Internet, these paths are representative as international and national (regional). The two paths also differ in QoS granularity, i.e. domains belonging to the path A3-R3-A4 are characterized by finer granularity than the ones from the path A1-R1-C-R2-A2.

TABLE 1: SPECIFICATION OF E2E SERVICE REQUESTS

\begin{tabular}{c|cccccc}
\hline \multirow{2}{*}{ Metric } & \multicolumn{7}{|c}{ Service request } \\
\cline { 2 - 7 } & $\mathbf{1}$ & $\mathbf{2}$ & $\mathbf{3}$ & $\mathbf{4}$ & $\mathbf{5}$ & $\mathbf{6}$ \\
\hline Delay (ms) & 150 & 200 & 400 & 400 & 600 & - \\
Jitter (ms) & 60 & 60 & 80 & - & - & - \\
PLR & $10^{-4}$ & $10^{-3}$ & $10^{-3}$ & $10^{-4}$ & $10^{-4}$ & $10^{-4}$ \\
\hline
\end{tabular}

\begin{tabular}{|c|c|c|c|c|c|}
\hline \multicolumn{6}{|c|}{ CLASSES) } \\
\hline $\begin{array}{c}\text { Domain path } \\
\text { A1-R1-C-R2-A2 }\end{array}$ & Class & $\begin{array}{c}\text { Delay } \\
(\mathrm{ms})\end{array}$ & $\begin{array}{c}\text { Jitter } \\
\text { (ms) }\end{array}$ & PLR & $\begin{array}{l}\text { Cost } \\
(\mathbf{M U})\end{array}$ \\
\hline \multirow{3}{*}{ A1 (access) } & 1 & 40 & 10 & $10^{-5}$ & 40 \\
\hline & 2 & 80 & 30 & $10^{-4}$ & 20 \\
\hline & 3 & 120 & - & $10^{-4}$ & 15 \\
\hline \multirow{4}{*}{ A2 (access) } & 1 & 20 & 15 & $10^{-6}$ & 55 \\
\hline & 2 & 50 & 20 & $10^{-5}$ & 30 \\
\hline & 3 & 70 & 30 & $5 \times 10^{-5}$ & 20 \\
\hline & 4 & 120 & - & $10^{-4}$ & 17 \\
\hline \multirow{2}{*}{ R1 (regional) } & 1 & 15 & 10 & $10^{-6}$ & 75 \\
\hline & 2 & 50 & 30 & $10^{-5}$ & 60 \\
\hline \multirow{2}{*}{ R2 (regional) } & 1 & 12 & 6 & $10^{-5}$ & 80 \\
\hline & 2 & - & - & $10^{-4}$ & 70 \\
\hline \multirow{3}{*}{$\mathrm{C}$ (continental) } & 1 & 45 & 5 & $10^{-5}$ & 90 \\
\hline & 2 & 100 & 15 & $5 \times 10^{-4}$ & 65 \\
\hline & 3 & 120 & 40 & $10^{-4}$ & 55 \\
\hline $\begin{array}{c}\text { Domain path } \\
\text { A3-R3-A4 }\end{array}$ & Class & $\begin{array}{c}\text { Delay } \\
(\mathrm{ms})\end{array}$ & $\begin{array}{c}\text { Jitter } \\
(\mathrm{ms})\end{array}$ & PLR & $\begin{array}{l}\text { Cost } \\
\text { (MU) }\end{array}$ \\
\hline \multirow{4}{*}{ A3 (access) } & 1 & 30 & 10 & $10^{-5}$ & 50 \\
\hline & 2 & 60 & 30 & $10^{-4}$ & 40 \\
\hline & 3 & 100 & - & $10^{-4}$ & 30 \\
\hline & 4 & - & - & $5 \times 10^{-3}$ & 15 \\
\hline \multirow{4}{*}{ A4 (access) } & 1 & 25 & 15 & $10^{-6}$ & 55 \\
\hline & 2 & 50 & 20 & $10^{-5}$ & 40 \\
\hline & 3 & 80 & 30 & $10^{-4}$ & 35 \\
\hline & 4 & 120 & - & $10^{-3}$ & 20 \\
\hline \multirow{3}{*}{ R3 (regional) } & 1 & 20 & 6 & $10^{-5}$ & 75 \\
\hline & 2 & 35 & 25 & $10^{-4}$ & 65 \\
\hline & 3 & 60 & - & $10^{-4}$ & 50 \\
\hline
\end{tabular}

TABLE 2: DOMAIN SPECIFICATION (HIERARCHY AND SERVICE

Comparison of E2E service performance in terms of delay, jitter and PLR along with overall interconnection cost is shown in Fig. 2 when class mappings are performed by the 3PIP and P-CMS. It is noticeable that 3PIP always conforms to the posed constraints. This is depicted in Fig. 2 (a)-(c) as 3PIP always selects classes that allow $D C>1$ for all posed QoS parameters. Conversely, P-CMS finds classes that are as close as possible to the perfect match, denoted as $D C=1$. Such an approach in mapping, although sometimes optimal, may create problems for services that require strict QoS values through SLA definition. Consequently, the 3PIP algorithm often provides overengineered class selection as can be noticed for service 
request 5 when offered E2E delay is nearly three times better than requested, whereas PLR for that same request is almost perfectly matched.

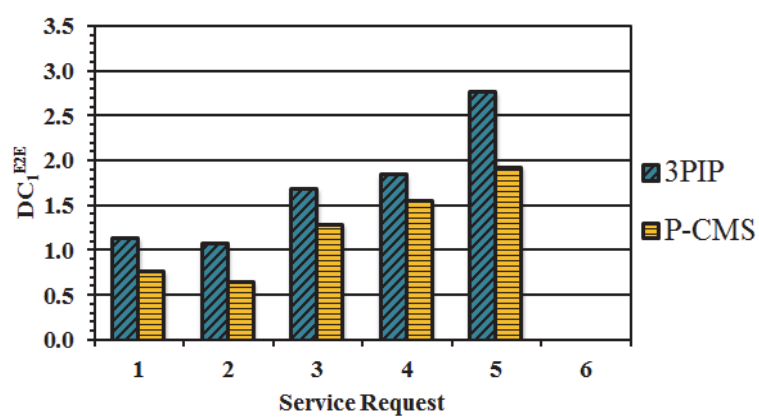

(a)

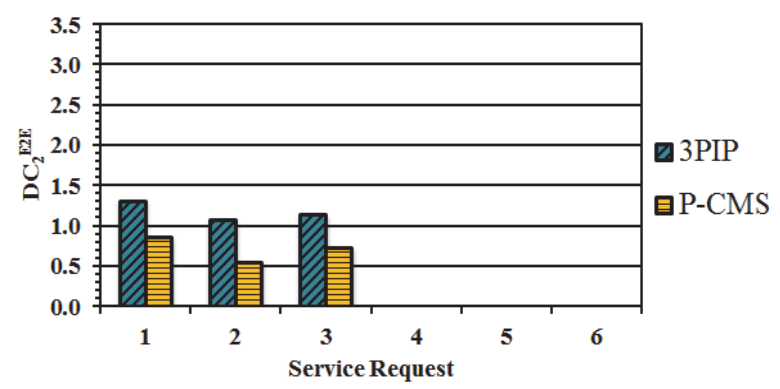

(b)

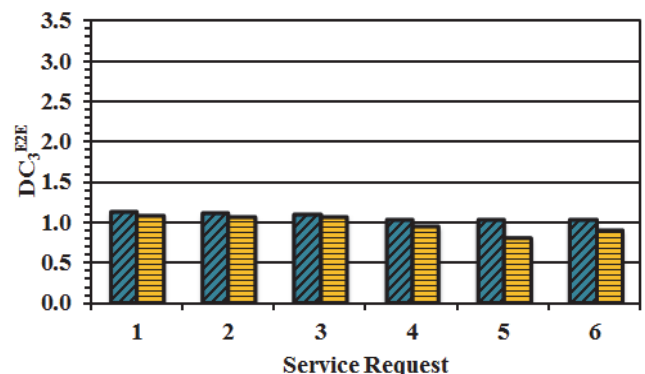

(c)

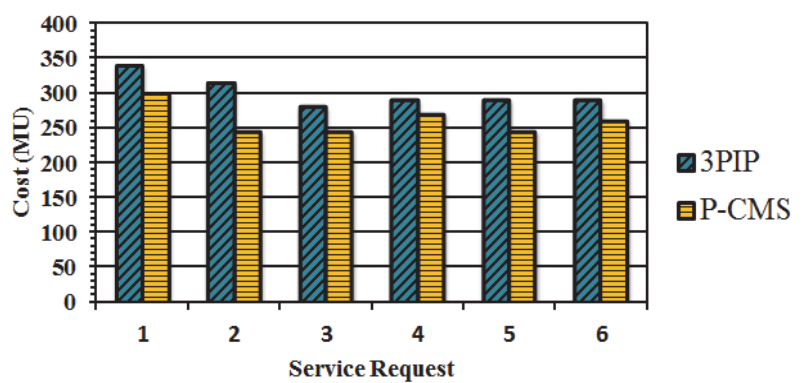

(d)

Fig. 2. Domain path A1-R1-C-R2-A2: (a) $D C_{1}{ }^{\mathrm{E} 2 \mathrm{E}}$ parameter (delay); (b) $D C_{2}{ }^{\mathrm{E} 2 \mathrm{E}}$ parameter (jitter); (c) $D C_{3}{ }^{\mathrm{E} 2 \mathrm{E}}$ parameter (PLR); (d) E2E service cost.

Costs per service class in Table 2 have been defined according to the domain hierarchy and the offered performance, bearing in mind guidelines in [20]. Fulfillment of all requested QoS metrics is reflected in a higher interconnection cost depicted in Fig. 2(d) for 3PIP mappings in comparison to P-CMS. This is a consequence of the selection of better classes. Although, 3PIP objective function drives low-priced mapping, P-CMS mappings yet have lower costs for all service requests as it targets classes with lower values of performance metrics.
Nevertheless, 3PIP approach is able to deliver E2E performance for services demanding strict QoS guarantees according to posed requirements, which at this point presents a trade-off between cost and exact mapping that delivers the required E2E QoS. An alternative approach is presented in [21] where a multi-objective approach is used if performance fulfillment is not possible.

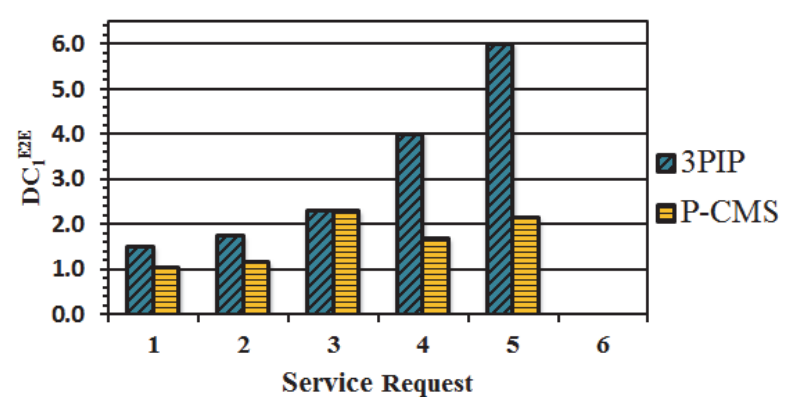

(a)

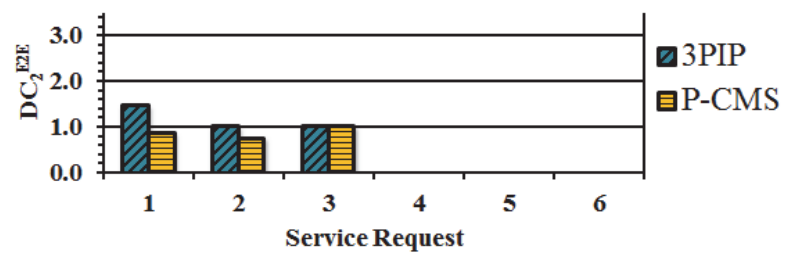

(b)

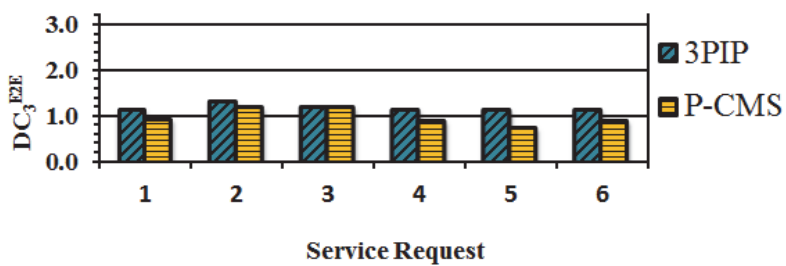

(c)

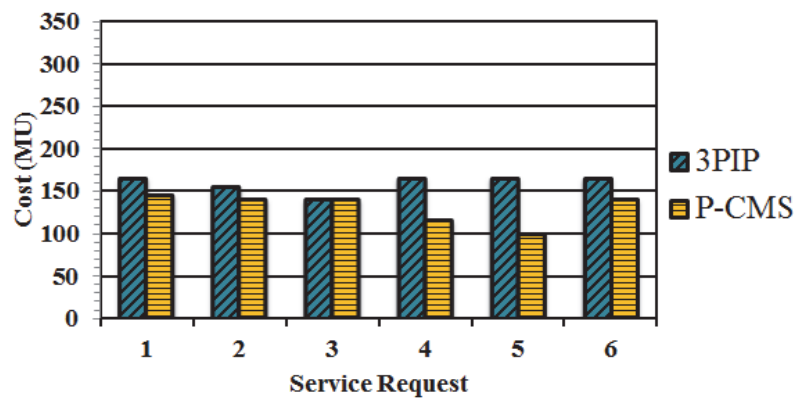

(d)

Fig. 3. Domain path A3-R3-A4: (a) $D C_{1}{ }^{\mathrm{E} 2 \mathrm{E}}$ parameter (delay); (b) $D C_{2}{ }^{\mathrm{E} 2 \mathrm{E}}$ parameter (jitter); (c) $D C_{3}{ }^{\mathrm{E} 2 \mathrm{E}}$ parameter (PLR); (d) E2E service cost.

As previously mentioned, the path A1-R1-C-R2-A2 contains domains with a wider range of service differentiation, which prompts for further investigation of service differentiation impact on class mapping. Consequently, we introduce another domain path A3-R3A4 with a finer QoS granularity. E2E performance and interconnection cost are depicted in Fig. 3, for 3PIP and PCMS algorithms. Aside from E2E delay, all 3PIP mappings are close to the perfect match. According to these results, we can draw the following conclusions. First, 3PIP does not make any difference regarding the number of domains on E2E path since it performs mapping on E2E level. Second, although service differentiation may affect 
the mapping accuracy in terms of the $D C$ parameter, it is important that classes are defined in a manner that makes fulfillment of E2E performance possible. For instance, although the second path A3-R3-A4 has better differentiation per domain, delay values defined in separate classes result in low latencies even for highly tolerant requests, e.g. service requests 4 and 5, as depicted in Fig. 3(a). Therefore, QoS granularity does not only refer to the number of defined service classes, but also to the range of performance metrics values.

Finally, E2E interconnection cost for the second path is lower in comparison to the first path, as a consequence that its domains belong to lower hierarchy levels (access and regional). It should be noted that P-CMS provides a lower interconnection cost for all service requests on the first path, and for majority of requests on the second path (except for service request 3 when both 3PIP and P-CMS select identical classes). This happens due to foundation for P-CMS class selection, i.e., the absence of E2E performance fulfillment in some cases. As a result of choosing classes with $D C$ values closest to 1, "worse" classes (in terms of QoS) are selected than with the 3PIP. Conversely, the 3PIP always delivers mapping with the least possible interconnection cost that enables full achievement of required E2E performance. Such a concept of 3PIP mapping enables the delivery of QoS for services such as voice and video, which highly rely on stringent QoS guarantees. For both paths, service requests 1 and 2 (specified to encompass strict QoS) are fulfilled when 3PIP mapping is implemented, thus highlighting the claim of being a convenient approach for services with stringent QoS demands.

\section{CONCLUSION AND FUTURE WORK}

Since the advantages of $3 \mathrm{P}$ service negotiation model become more evident as multi-domain services with $\mathrm{E} 2 \mathrm{E}$ QoS requirements rapidly emerge, a service class mapping algorithm that is able to address mapping in dynamic multi-domain scenarios needs to be introduced. To this end, in this paper we have proposed the 3PIP algorithm that accomplishes cost effective mapping. This contributes to increase of the projected profit, while at the same time delivering service which satisfies E2E QoS requirements. The proposal is a notable alternative to the existing $3 \mathrm{P}$ mapping schemes, particularly regarding services with stringent QoS requirements. Our future work should encompass investigation of QoS routing via a 3P agent along with the integration of the proposed class mapping scheme.

\section{REFERENCES}

[1] M. E. M. Campista, M. G. Rubinstein, I. M. Moraes, L. H. M. K. Costa, and O. C. M. B. Duarte, "Challenges and research directions for the future internetworking", IEEE Communications Surveys and Tutorials, vol. 16, no. 2, Second Quarter 2014, pp. 1050-1079.

[2] P. Levis and M. Boucadair, Considerations of provider-to-provider agreements for Internet-scale quality of service (QoS), IETF RFC 5160, 2008.

[3] P. Jacobs and B. Davie, "Technical challenges in the delivery of interprovider QoS”, IEEE Communications Magazine, vol. 43, no. 6, June 2005, pp. 112-118.

[4] J. Wulf, F. Limbach, and R. Zarnekow, "Analysis of QoS platform cooperation strategies", in Proc. 21st European Regional ITS Conference, Copenhagen, Denmark, 2010, 17 pages.

[5] J. Zhao, J. Guan, C. Xu, Wei Su, and H. Zhang, "Peering strategic game models for interdependent ISPs in content centric Internet", The Scientific World Journal, vol. 2013, 10 pages, 2013.

[6] M. Ángeles Callejo-Rodriguez and J. Enriquez-Gabeiras, "Bridging the standardization gap to provide QoS in current NGN architectures", IEEE Communications Magazine, vol. 46, no. 10, Oct. 2008, pp. 132-137.

[7] Network performance objectives for IP-based services, ITU-T Recommendation Y.1541, 2011.

[8] E. Mingozzi, G. Stea, M. A. Callejo-Rodríguez et al., "EuQoS: End-to-end quality of service over heterogeneous networks", Computer Communications, vol. 32, no. 12, July 2009, pp. 13551370 .

[9] M. Stojanovic, S. Bostjancic Rakas, and V. Acimovic-Raspopovic "End-to-end quality of service specification and mapping: the third party approach", Computer Communications, vol. 33, no. 11, July 2010, pp.1354-1368.

[10] M. Stojanovic and S. Bostjancic Rakas, "Policies for allocating performance impairment budgets among multiple IP providers". AEÜ-International Journal of Electronics and Communications, vol. 67, no.3, Mar. 2013, pp. 206-216.

[11] Framework for achieving end-to-end IP performance objectives, ITU-T Recommendation Y.1542, 2010.

[12] C. A. Kamienski and D. Sadok, "The case of interdomain dynamic QoS-based service negotiation in the internet", Computer Communications, vol. 27, no. 7, May 2004, pp. 622-637.

[13] V. Sarangan and J. C. Chen, "Comparative study of protocols for dynamic service negotiation in the next-generation Internet", IEEE Communications Magazine, vol. 44, no. 3, Mar. 2006, pp. 151-156.

[14] J. Manner, R. Bless, J. Loughney, and E. Davies, Using and extending the NSIS protocol family, IETF RFC 5978, 2010.

[15] B. Mali, N. Ninkovic, M. Stojanovic and G. Savic, "Service class mapping based on integer programming algorithm in the third party agent", in Proc. of the 22nd Telecommunications Forum TELFOR 2014, Belgrade, Serbia, 2014, pp. 170-173.

[16] B. Korte and J. Vygen, Combinatorial Optimization: Theory and Algorithms, 5th edition. Springer Berlin Heidelberg, 2012, ch. 5.

[17] P. H. Williams, Model Building in Mathematical Programming, 5thedition. Chichester, UK: John Wiley \& Sons, 2013, ch. 3.

[18] R. A. Dourado, L. N. Sampaio, and J. A. SuruagyMonteiro, "On the composition of performance metrics in multi-domain networks", IEEE Communications Magazine, vol. 51, no. 11, Nov. 2013, pp. 72-77.

[19] W. L. Winston, Operations Research: Applications and Algorithms, 4th edition. Belmont, CA: Duxbury Press, 2003, ch. 9.

[20] M. Stojanovic, A. Kostic-Ljubisavljevic, and V. Radonjic Djogatovic, "SLA-controlled inter-connection charging in next generation networks", Computer Networks, vol. 57, no. 11, Aug. 2013, pp. 2374-2394.

[21] Nemanja M. Ninkovic, Bozidar J. Mali, Mirjana D. Stojanovic and Gordana I. Savic, "Multi-Objective Third-party Approach for Service Class Mapping among Multiple Providers in the Internet", Elektronika IR Elektrotechnika, Vol. 21, No. 2, 2015, pp. 80-84. 